\title{
A long-term influence of anthropogenic alkalization on molluscs biodiversity in an area affected by cement industry, Świętokrzyskie Mountains, South-Central Poland
}

\author{
Jadwiga Anna Barga-Więcławska ${ }^{1}$ Anna Świercz ${ }^{2 *}$ \\ Jan Kochanowski University, Poland \\ Faculty of Environmental Protection and Management \\ ${ }^{1}$ Institute of Biology \\ Department of Zoology \\ ${ }^{2}$ Department of Soil and Cultural Landscape Conservation \\ *Corresponding author's e-mail: swierczag@poczta.onet.pl
}

\begin{abstract}
Keywords: malacofauna, cement industry, biodiversity, soil properties, Świętokrzyskie Mountains, South-Central Poland.

Abstract: The presented paper reports data from malacological and pedological studies carried out at sites representing diverse biotopes (beech wood, coniferous forest, and meadow) located $2 \mathrm{~km}$ away from the Dyckerhoff Cement Plant in Sitkówka-Nowiny in 1992 and in 2008-2009. The studies aimed to determine physicochemical properties of soils exposed to cement and limestone dust emission and to identify composition of snail communities inhabiting three different biotopes in relation to physicochemical properties of soils, and to grasp the dynamics of the alkalization-dependent changes in physicochemical properties of soils and their impact on the composition and ecological structure of malacofauna.
\end{abstract}

\section{Introduction}

Soil alkalization is a phenomenon consisting in a change in its originally acidic $\mathrm{pH}$ into alkaline $\mathrm{pH}$ due to ionic exchange within sorption complex. Most often this process is caused by liming, i.e. purposeful soil fertilization, by salinization or by local carbonate dust emission of anthropogenic origin (e.g. Derome et al. 1986, Świercz 1995, Świercz 2005, Ade-Ademilua and Obalola 2008, Addo et al. 2013). Cement and lime industry emits large amounts of dust which, though included into non-toxic emission, disturbs stability of the environment (Mandre and Lukjanowa 2011, Simpson et al. 2012). Environmental effect of cement-lime industry, involving changes in species composition of animal and plant populations, alterations in physicochemical properties of soils, impairment of assimilation organs of plants and tree bark, have been addressed in numerous articles (Ade-Ademilua and Obalola 2008, Iqbal and Shafig 2001, Simpson et al. 2012, Świercz 2006, Tervahattu et all 2001, Jaworska et al. 2010). The area called "White Mining District" in the Świętokrzyskie Voivodeship has been at particular risk of alkalization due to tremendous development of mining and processing of carbonate rocks (Kozłowski 2013, Łubek 2010, Świercz 2010). The main alkaline dust emitters in his region include: Dyckerhoff Cement Plant in Sitkówka-Nowiny, Lime Industry Works in Trzuskawica, and Lafarge Cement Plant in Małogoszcz. Since 1991 dust and gas emission has been successively decreasing (even 40-fold) and the reduction of air pollution resulted from modernization of production lines and lowered cement output (Świercz 2006, 2010). In the period from 1983 to 2011 an average annual dust emission only from the cement plant in Sitkówka-Nowiny amounted to $4000 \mathrm{Mg}$ (with the maximum of $24,600 \mathrm{Mg}$ in 1984) while today it remains at the level of $100 \mathrm{Mg}$ per year and does not exceed Ambient Air Quality Standards. These changes in dust deposition in soil strongly influenced organisms inhabiting these terrains (Iqbal and Shafig 2001, Simpson at. al. 2012, Świercz 2005). Snails belong to particularly sensitive bioindicators. They are widespread in terrestrial and aquatic environments, can be easily gathered, are characterized by specific requirements for $\mathrm{CaCO}_{3}$ in soil and rapidly react to humidity changes (Barga-Więcłwska 1997, 2005, Kappes 2006, Kappes et al. 2006). They react to environmental stress with changes in composition of snail associations and snail density. Snails have a short lifespan which is significant for immediate recording of changes and frequent repetition of surveys. Snails are an important component of edaphone and their occurrence depends on quality and physicochemical characteristics of soils (Beeby and Richmond 2004, Martin and Sommer 2004). According to Hylander (2011), calcium content and soil $\mathrm{pH}$ are the key factors critical for species composition and density of snails in a given habitat. Thus, these features were decisive for the choice of this group of organisms for studies on alkalization of habitats.

The present paper contains results of malacological and pedological studies conducted in the years 1992-1994 
and repeated in 2000-2001 and then in 2008-2009 at three sites representing different biotopes (hornbeam-beech woods, coniferous forest, and meadow) located in the vicinity of the Malik Hill $2 \mathrm{~km}$ away from the Dycerhoff Cement Plant in Sitkówka-Nowiny. The studies conducted in the 1990s describe habitats and malacofauna after a 30-year heavy deposition of lime-cement dust in soils at the maximal alkalization, and then changes that occurred with lowering of dust deposition in the following 11 and 16 years are characterized.

The studies aimed to:

- determine physicochemical properties of soils exposed to lime and cement dust deposition;

- determine composition of snail communities inhabiting three different biotopes (at 3 sites) in dependence on changing physiocochemical properties of soils,

- determine the rate of changes in specific soil horizons and their impact on malacofauna composition and ecological structure.

\section{Materials and methods}

Soil studies were conducted at 3 sites in 1992 and 2009. In total 6 soil pits were dug to a depth of $200 \mathrm{~cm}$ and samples for laboratory studies were collected from the identified soil horizons. The samples were air-dried and the following soil characteristics were determined: particle size distribution in skeletal fractions and sand fraction using a sieve method, and in dust and fine fraction using an aerometric method of Casagrande as modified by Prószyński, $\mathrm{pH}$ in $\mathrm{H}_{2} \mathrm{O}$ and $1 \mathrm{M}$ $\mathrm{KCl}$ using a potentiometric method, $\mathrm{CaCO}_{3}$ content using a volumetric method of Sheibler, organic carbon content in mineral horizons by the method of Tiurin and in organic horizons by the method of Alten, total nitrogen content after mineralization by the method of Kjeldahl using an Kjeltec Auto 1030 autoanalyzer, hydrolytic acidity according to Kappen in $0.5 \mathrm{M}$ solution of $\mathrm{Ca}\left(\mathrm{CH}_{3} \mathrm{COO}\right)_{2}$, sum of exchangeable bases, based on the equation: $\left.\mathrm{S} 1=\sum \mathrm{Ca}^{2+}, \mathrm{Mg}^{2+}, \mathrm{K}^{+}, \mathrm{Na}^{+}\right)$in $1 \mathrm{M} \mathrm{CH}_{3} \mathrm{COONH}_{4}$ (for carbonate-free samples), and in $0.5 \mathrm{M}$ $\mathrm{NH}_{4} \mathrm{Cl} \mathrm{pH} 8.2$ (for carbonate-containing samples) (Ostrowska et al. 1991). Based on the sum of bases (S1) and hydrolytic acidity (Hh), sorption capacity (T) and soil base saturation (V) values were calculated.

Malacofauna studies were conducted near soil pits in the periods: 1993-1994, 2000-2001 and 2008-2009. Snails were sampled from soils and plants in spring (June 1-10) and in autumn (September 20-30) of each study year. A standard absolute method of Oekland (1930) was used which assumes that a random collection of 16 soil samples from a phytosociological plot with a metal frame measuring $25 \mathrm{~cm} \times 25 \mathrm{~cm}$ to a depth of $3-5 \mathrm{~cm}$ corresponds to examining an area of $1 \mathrm{~m}^{2}$. The quantitative method was complemented with a qualitative method based on visual examination of plant leaves, rocks and rotting tree stamps. In the laboratory, the soil samples collected for snail studies were examined manually under a binocular magnifying glass. Specimens collected each year were preserved in archives. Calculations were based on live snails. Ecological groups were distinguished according to Ložek (1964). Ecological structure of molluscs was presented in a Table and by circular malacospectra according to Alexandrowicz (1987) and Alexandrowicz and Alexandrowicz (2011).

\section{Study sites}

The study sites were located on the Malik Hill (Fig. 1). The Malik Hill is a karsted fault block (275 m above see level) located in the south-western part of the Świętokrzyskie Mountains on the northern slopes of the Bolechowice Range (Fig. 2). It is built mostly of Middle Devonian amphiporous limestone covered on the hilltop with a thin clayey rock-mantle which on the slopes is interlocked with deep layered Pleistocene sands and gravel (Kowalkowski and Świercz 1993, Świercz 2010), turning into alluvial formations of the Bobrzyczka River in the east. In 1993 Environmental Monitoring Station was established there to analyze the alkaline emission-induced changes in the geoecosystem.

Site I (hornbeam-beech forest) was located on the hilltop plateau of the Malik Hill (250-260 m above sea level) in a hornbeam-beech forest developing on rusty proper rendzinas. The forest that grew in that terrain could be classified as a degenerative form of subcontinental Tilio-Carpinetum association (Tracz 1962) with a complex of species characteristic of the alliance Carpinion betuli, the order Fagetalia silvaticae and the class Querco-Fagetea (Świercz 1995). The main forest-forming species was even-aged secondary pine tree. The layer $\mathrm{A}_{2}$ with $80 \%$ density was composed of underbrush with Carpinus betulus, Fagus sylvatica, Corylus avellana, Acer pseudoplatanus, Quercus petraea. The layer $b$ with $50 \%$ density was formed by Corylus avellana, Fagus sylvatica, Lonicera xylosteum, Abies alba. The herb layer with $80 \%$ density was built mostly of Asarum europaeum, Lamiastrum galeobdolon, Mercurialis perennis, Viola reichenbachiana, Hepatica nobilis, Carex digitata, Cruciata glabra. The moss layer was scarce. Forty three species were identified in the phytosociological record. The site was shady and wet. Carbonate rock outcrops and deciduous leaf litter created advantageous environment for snail refuges.

Site II (coniferous forest) was located on a subslope flattening of the Malik Hill in the coniferous forest growing on red podzolic soil. The forest was a degenerative form of pine forest Peucedano-Pinetum (W.Mat.1962, W. Mat and J. Mat. 1973). Even-aged 70-year secondary pine tree was the main forest-forming species. The layer A had a density of $75 \%$ while density of bush layer was $20 \%$ and contained Quercus robur, Sorbus aucuparia, Juniperus communis, Frangula alnus. An undergrowth with $60 \%$ density was composed of Festuca ovina, Fragaria vesca, Majanthemum bifolium, Chamaecytisus ratisbonensis, and sporadically Vaccinium myrtillus, V. vitis idaea and other coniferous forest species occurred due to the presence of calcium of anthropogenic origin in soil. The moss layer was well-developed and covered $90 \%$ of the area. The main species were: Hylocomnium splendens, Scleropodium purum, Mnium affine, Brachythecium salebrosum. The site was light, quite dry with scarce refuges for snails.

Site III (meadow) was located on a flooded terrace of the Bobrzyczka River below the Malik Hill in the community (meadow type) which grew on ground-water gley soil. Plant community was a semi-natural fresh wet meadow with species composition corresponding to Cirsio-Polygonetum (R.Tx.1951) and remained under anthropogenic pressure. The meadow was bordered by willow-alder brushwood on the south. The site was warm, humid, periodically wet, creating good condition for the development of snails. 


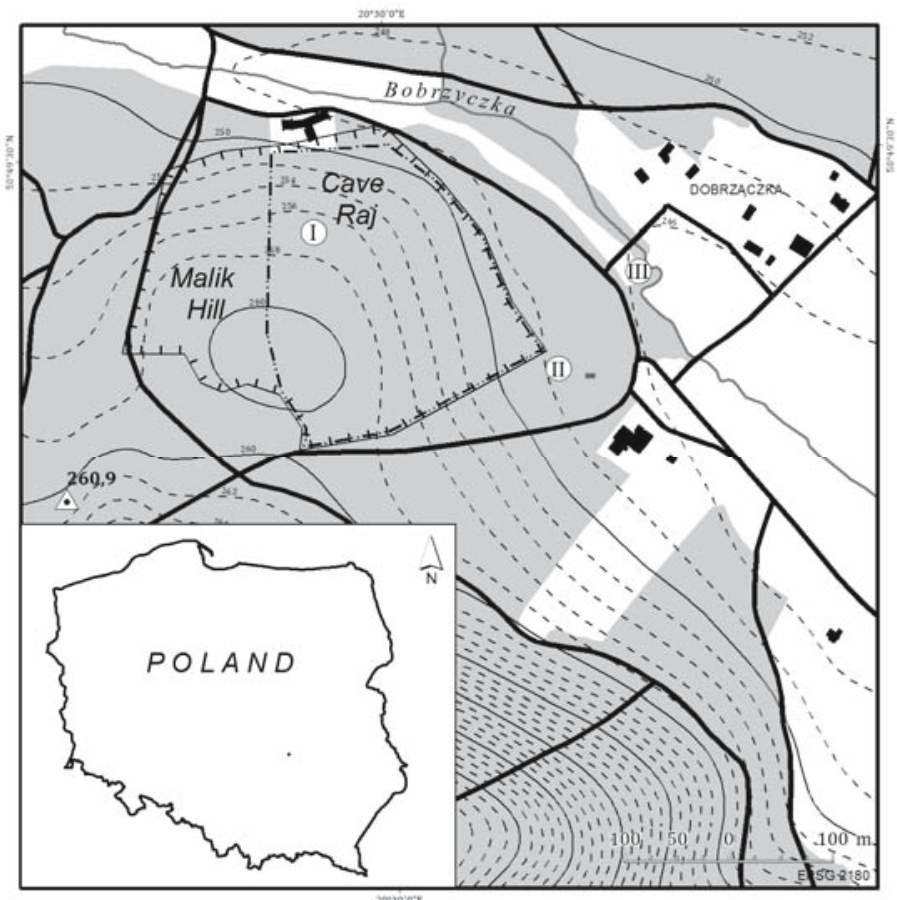

Fig. 1. Localization of study area: I Tilio-Carpinetum, hornbeam-beech forest; II Peucedano-Pinetum, coniferous forest; III Cirsio-Polygonetum, meadow

Throughout the years 1992-2009, meteorological conditions at the study sites changed negligibly. Data from the Malik Environmental Monitoring Station indicated that annual precipitation was from $538 \mathrm{~mm}$ (in 2009) to $617 \mathrm{~mm}$ (in 2001) while annual air temperatures ranged from $8.0^{\circ} \mathrm{C}$ (in 2001) to $9.1^{\circ} \mathrm{C}$ (Kozłowski 2013).

No significant differences in vascular plant composition at the study sites were observed throughout the study period. Only at site II in coniferous forest, the development of new coniferous forest species, such as Melampyrum pratense $(+)$ oraz Trientalis europaea $(+)$, absent in 1992, was noted in 2009 .

\section{Results}

Physicochemical properties of soils under study were diverse and modified molluscan fauna (Tab. 1). At site I (hornbeam-beech woods), the alkalized soils belonged to proper rendzinas developed from dark red (2,5 YR 4/6), rocky weathered loams containing $51 \%$ of fine particulate matter and $14 \%$ of dust fraction with a considerable admixture of sand, overlaying shallowly Middle Devonian limestone. Soil $\mathrm{pH}_{\mathrm{KCl}}$ ranged from 6.50 to 7.30 in $1992 \mathrm{r}$. and decreased slightly to the level from 6.22 to 7.05 in 2009 .

Lower values of $\mathrm{pH}_{\mathrm{KCl}}$ were characteristic of the parent rock horizon Cca. This was a specific phenomenon for this area and could be linked to the processes forming calciumfree mantle rock of terra rossa type in the Świętokrzyskie Mountains (Kowalkowski and Świercz 1993). The occurrence of malacofauna is dependent on calcium content in substratum. Carbonate content in the soil under study amounted to max. $4.13 \%$ in 1992 r. and to $3.22 \%$ in 2009 r. Enrichment of litter and organic-mineral horizons in carbonates was of distinct anthropogenic origin. Hydrolytic acidity in the studied rendzina soil was low and ranged from 2.11 to $0.1 \mathrm{cmol}(+) / \mathrm{kg}$. The base saturation of sorption complex exceeded $95 \%$ while the sum of bases was in the range of $60.11-14.45 \mathrm{cmol}(+) / \mathrm{kg}$ in 1992 and 57.06-13.97 in 2009 with exchangeable calcium being the dominating element. Carbon and nitrogen total content was typical of rendzinas in Poland (Brożek and Zwydak 2003) and did not significantly change within the study period.

At site I (hornbeam-beech forest) 31 snail species were recorded in 1993-1994. Indicator species comprised two euryecological species Euconulus fulvus $\mathrm{C}_{5} \mathrm{D}_{5}$ and Punctum pygmaeum $\mathrm{C}_{4} \mathrm{D}_{5}$ and forest species: Acanthinula aculeata $\mathrm{C}_{4} \mathrm{D}_{4}$ and Aegapinella pura $\mathrm{C}_{4} \mathrm{D}_{4}$ (Tab.2). At the same site, 26 snail species were identified in 2000-2001, with indicator species the same as in the earlier period: E. fulvus $\mathrm{C}_{5} \mathrm{D}_{5}$ and P. pygmaeum $\mathrm{C}_{5} \mathrm{D}_{4}$. The rank of the forest species $A$. aculeata $\mathrm{C}_{4} \mathrm{D}_{3}$ and $A$. pura $\mathrm{C}_{4} \mathrm{D}_{3}$ in the assemblage declined since they became accessory species. In the 2008-2009 survey, 29 species were recorded at this site with two bush-preferring species, rare in the Świętokrzyskie Mountains, Vitrea crystallina $\mathrm{C}_{5} \mathrm{D}_{4}$ and Oxychillus allarius $\mathrm{C}_{5} \mathrm{D}_{4}$ being indicator species. Noteworthy was a decline in the rank of species of shady habitats and a decrease in rank of taxons typical of natural forests preferring shade and medium humidity, and advancement of species more tolerant to light and humidity.

Within the space of 16 years the number of companion species was high and remained at the same level. Based on the studies in the Chęciński Region, a large number of companion species is characteristic of snail communities occurring in habitats developing of carbonate rocks of anthropogenic origin (Barga-Więcławska 1997).

Analysis of ecological structure of snail communities was the next element helping to characterize the changes in malacocenoses exposed to variable dust deposition in soils (Tab. 3, Fig.2). 
Table 1. Some chemical properties of the investigated soils in the 1992-2009 period

\begin{tabular}{|c|c|c|c|c|c|c|c|c|c|c|c|c|}
\hline \multirow{2}{*}{$\begin{array}{l}\text { Study } \\
\text { area }\end{array}$} & \multirow{2}{*}{ Horizons } & \multirow{2}{*}{$\begin{array}{c}\text { Dept } \\
\text { in cm } \\
{[13]}\end{array}$} & \multirow{2}{*}{$\begin{array}{c}\mathrm{pH} \\
\mathrm{H}_{2} \mathrm{O}\end{array}$} & \multirow{2}{*}{$\begin{array}{l}\mathrm{pH} \\
\mathrm{KCl}\end{array}$} & $\mathrm{CaCO}_{3}$ & C org. & $\mathrm{N}$ og. & \multirow[t]{2}{*}{ C:N } & $\mathrm{Hh}$ & S1 & $\mathrm{T}$ & \multirow{2}{*}{$\begin{array}{l}\mathrm{V} \\
\%\end{array}$} \\
\hline & & & & & \multicolumn{3}{|c|}{ [\%] } & & \multicolumn{3}{|c|}{$[\mathrm{cmol}(+) / \mathrm{kg}]$} & \\
\hline \multicolumn{13}{|c|}{$1992 \mathrm{r}}$. \\
\hline \multirow[t]{6}{*}{ I } & Ol & $0-1$ & 7.40 & 7.15 & 0.60 & 40.24 & 1.32 & 30.5 & 2.11 & 46.12 & 48.23 & 95.63 \\
\hline & Ofh & $1-2$ & 7.53 & 7.30 & 3.15 & 20.45 & 0.92 & 22.2 & 0.88 & 60.11 & 60.99 & 98.56 \\
\hline & Ah & $2-6$ & 7.35 & 6.70 & 4.13 & 9.11 & 1.11 & 8.2 & 1.23 & 36.16 & 37.39 & 96.71 \\
\hline & $\mathrm{Bbr}$ & $6-25$ & 7.10 & 6.50 & 3.23 & 0.96 & 0.09 & 10.7 & 0.89 & 18.26 & 19.15 & 95.35 \\
\hline & BbrCca & $25-40$ & 7.00 & 6.63 & 2.62 & 0.34 & 0.03 & 11.3 & 0.55 & 14.45 & 15 & 96.33 \\
\hline & Cca & $40-70$ & 7.00 & 6.70 & 1.12 & - & - & - & 0.10 & 17.11 & 17.21 & 99.42 \\
\hline \multirow[t]{6}{*}{ II } & $\mathrm{OI}$ & $0-1$ & 6.95 & 6.55 & 0.34 & 37.60 & 1.12 & 33.6 & 6.90 & 56.91 & 63.81 & 89.19 \\
\hline & Ofh & $1-3$ & 7.00 & 6.74 & 3.24 & 19.38 & 0.72 & 26.9 & 1.85 & 39.11 & 40.96 & 95.48 \\
\hline & AEes & $3-12$ & 6.55 & 6.30 & 1.65 & 0.92 & 0.23 & 4.0 & 0.87 & 14.53 & 15.4 & 94.35 \\
\hline & BvBfe & $12-45$ & 6.43 & 6.10 & 0.70 & 0.43 & 0.07 & 6.1 & 0.43 & 5.15 & 5.58 & 92.29 \\
\hline & $\mathrm{Bv}$ & $45-80$ & 6.33 & 6.00 & 0.30 & 0.11 & 0.02 & 5.5 & 0.61 & 3.17 & 3.78 & 83.86 \\
\hline & $C$ & $80-120$ & 6.10 & 5.90 & 0.0 & - & - & - & 0.56 & 3.10 & 3.66 & 84.70 \\
\hline \multirow[t]{4}{*}{ III } & 0 & $0-2$ & 6.91 & 6.64 & 0.92 & 36.88 & 1.42 & 26.0 & 9.12 & 44.21 & 53.33 & 82.90 \\
\hline & Ah & $2-15$ & 6.45 & 6.32 & 1.35 & 2.80 & 0.29 & 9.7 & 12.11 & 16.13 & 28.24 & 57.12 \\
\hline & $\mathrm{Gr}$ & $15-60$ & 5.57 & 5.45 & 0.0 & 0.74 & 0.09 & 8.2 & 3.72 & 18.11 & 21.83 & 82.96 \\
\hline & CGr & $60-100$ & 5.51 & 5.35 & 0.0 & 0.26 & 0.05 & 5.2 & 4.11 & 14.36 & 18.47 & 77.75 \\
\hline \multicolumn{13}{|c|}{2009 r. } \\
\hline \multirow[t]{6}{*}{ I } & Ol & $0-1$ & 7.00 & 6.95 & 0.20 & 39.07 & 1.36 & 28.7 & 2.19 & 39.21 & 41.4 & 94.71 \\
\hline & Ofh & $1-2$ & 7.30 & 7.05 & 2.91 & 18.35 & 0.98 & 18.7 & 1.07 & 57.06 & 58.13 & 98.16 \\
\hline & Ah & $2-6$ & 6.91 & 6.22 & 3.22 & 8.17 & 1.08 & 7.6 & 1.54 & 32.14 & 33.68 & 95.43 \\
\hline & $\mathrm{Bbr}$ & $6-25$ & 6.87 & 6.52 & 2.33 & 0.94 & 0.07 & 13.4 & 0.83 & 19.16 & 19.99 & 95.85 \\
\hline & BbrCca & $25-40$ & 6.97 & 6.54 & 1.97 & 0.21 & 0.03 & 7.0 & 0.59 & 13.97 & 14.56 & 95.95 \\
\hline & Cca & $40-70$ & 7.07 & 6.75 & 1.27 & - & - & - & 0.08 & 16.22 & 16.3 & 99.51 \\
\hline \multirow[t]{6}{*}{ II } & Ol & $0-1$ & 6.76 & 6.44 & 0.29 & 38.11 & 1.11 & 34.3 & 7.08 & 51.21 & 58.29 & 87.85 \\
\hline & Ofh & $1-3$ & 6.92 & 6.63 & 2.54 & 17.48 & 0.82 & 21.3 & 1.99 & 32.81 & 34.8 & 94.28 \\
\hline & AEes & $3-12$ & 6.61 & 6.41 & 1.31 & 0.87 & 0.23 & 3.8 & 0.96 & 12.49 & 13.45 & 92.86 \\
\hline & BvBfe & $12-45$ & 6.39 & 6.00 & 0.53 & 0.45 & 0.06 & 7.5 & 0.63 & 3.18 & 3.81 & 83.46 \\
\hline & $\mathrm{Bv}$ & $45-80$ & 5.97 & 5.88 & 0.0 & 0.31 & 0.03 & 10.3 & 0.59 & 2.76 & 3.35 & 82.39 \\
\hline & C & $80-120$ & 5.38 & 5.22 & 0.0 & - & - & - & 0.61 & 2.66 & 3.27 & 81.35 \\
\hline \multirow[t]{4}{*}{ III } & 0 & $0-1$ & 6.56 & 6.43 & 0.54 & 37.81 & 1.53 & 24.7 & 10.07 & 41.05 & 51.12 & 80.30 \\
\hline & Ah & $1-15$ & 6.35 & 6.28 & 1.12 & 2.91 & 0.31 & 9.4 & 13.12 & 14.33 & 27.45 & 52.20 \\
\hline & $\mathrm{Gr}$ & $15-60$ & 5.51 & 5.37 & 0.0 & 0.62 & 0.08 & 7.8 & 3.41 & 17.18 & 20.59 & 83.44 \\
\hline & CGr & $60-100$ & 5.42 & 5.12 & 0.0 & 0.21 & 0.04 & 5.3 & 3.93 & 10.16 & 14.09 & 72.11 \\
\hline
\end{tabular}

In 1993-1994, the percentage of mesophilous and forest snail species (E1, E2, E3, E7 and E8) was high and amounted to $74 \%$, of which $23 \%$ were forest species (Fig. 2). This site was abundantly populated by the forest species $A$. aculeata, A. pura and Perforatella incarnata. In addition, the species Isognomostoma isognomostoma occurring in natural montane forests but rare in the Świętokrzyskie Mountains was also found. The disturbance of natural character of the hornbeambeech forest was evidenced by a scanty population of only one forest species Cochlodina laminata requiring good humidity and living on rotting wood and in leaf litter of deciduous forests. A high level of dust pollution in the study area lasting for 30 years abruptly limited populations of Clausiliidae inhabiting trees and litter. On the other hand, relatively abundant populations of hygrophilous species Columella edentula and Nesovitrea petronella and species preferring wet habitats Zonitoides nitidus and Perforatella rubiginosa are indicative of good humidity conditions of microhabitats in the hornbeam-beech forest. Species of dry meadows were scanty and rare $(E 5,16 \%)$. This can be explained by very confined changes in environmental conditions of microhabitats at the forest floor dependent on dust deposition and tree species composition (canopies of deciduous trees keep larger dust amounts on leaves than coniferous trees). 


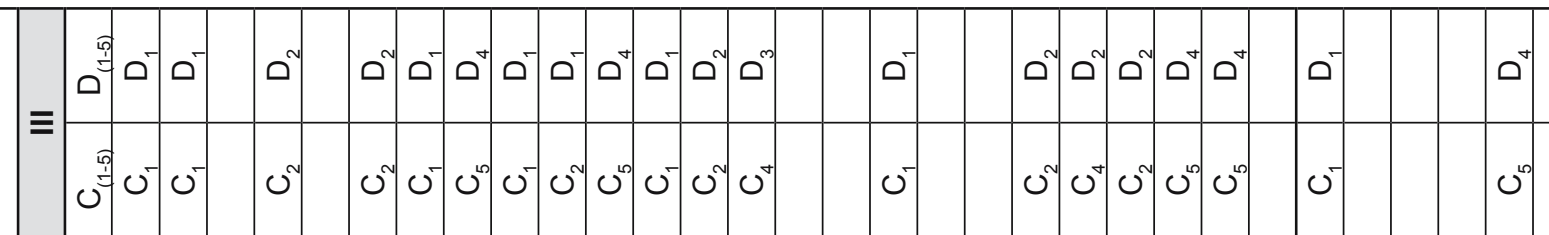

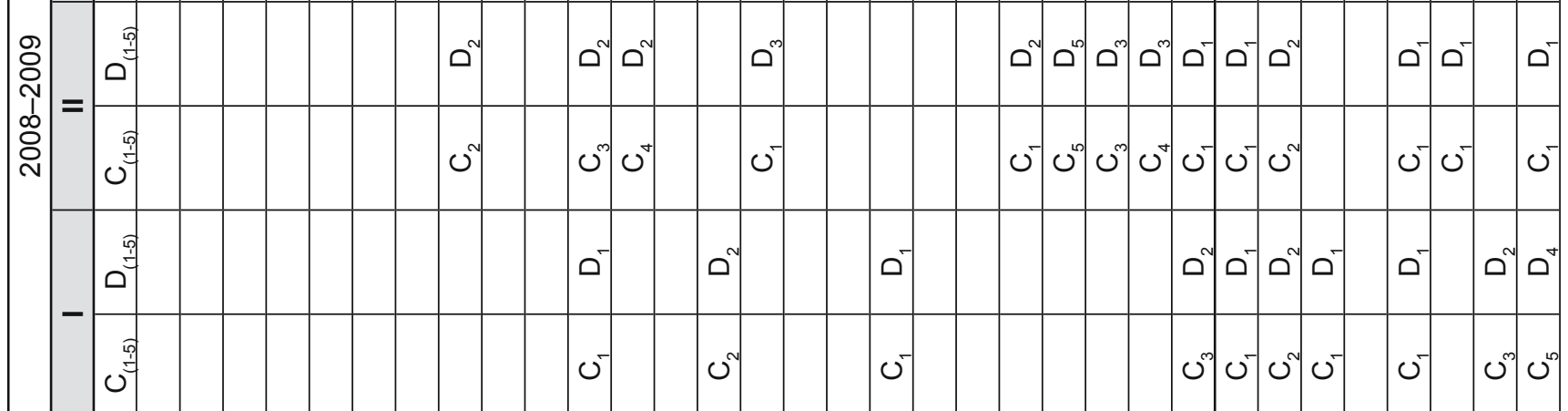

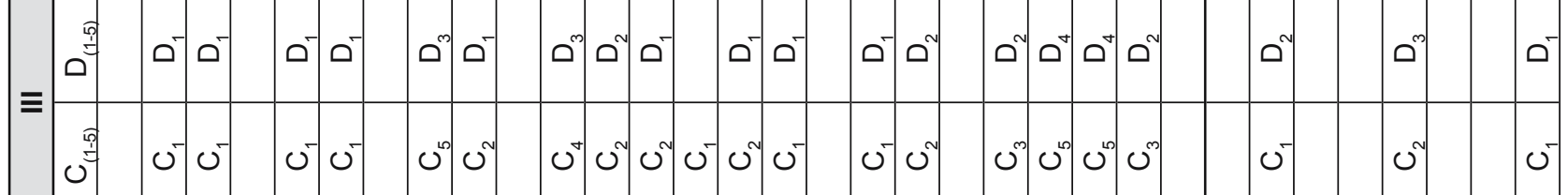

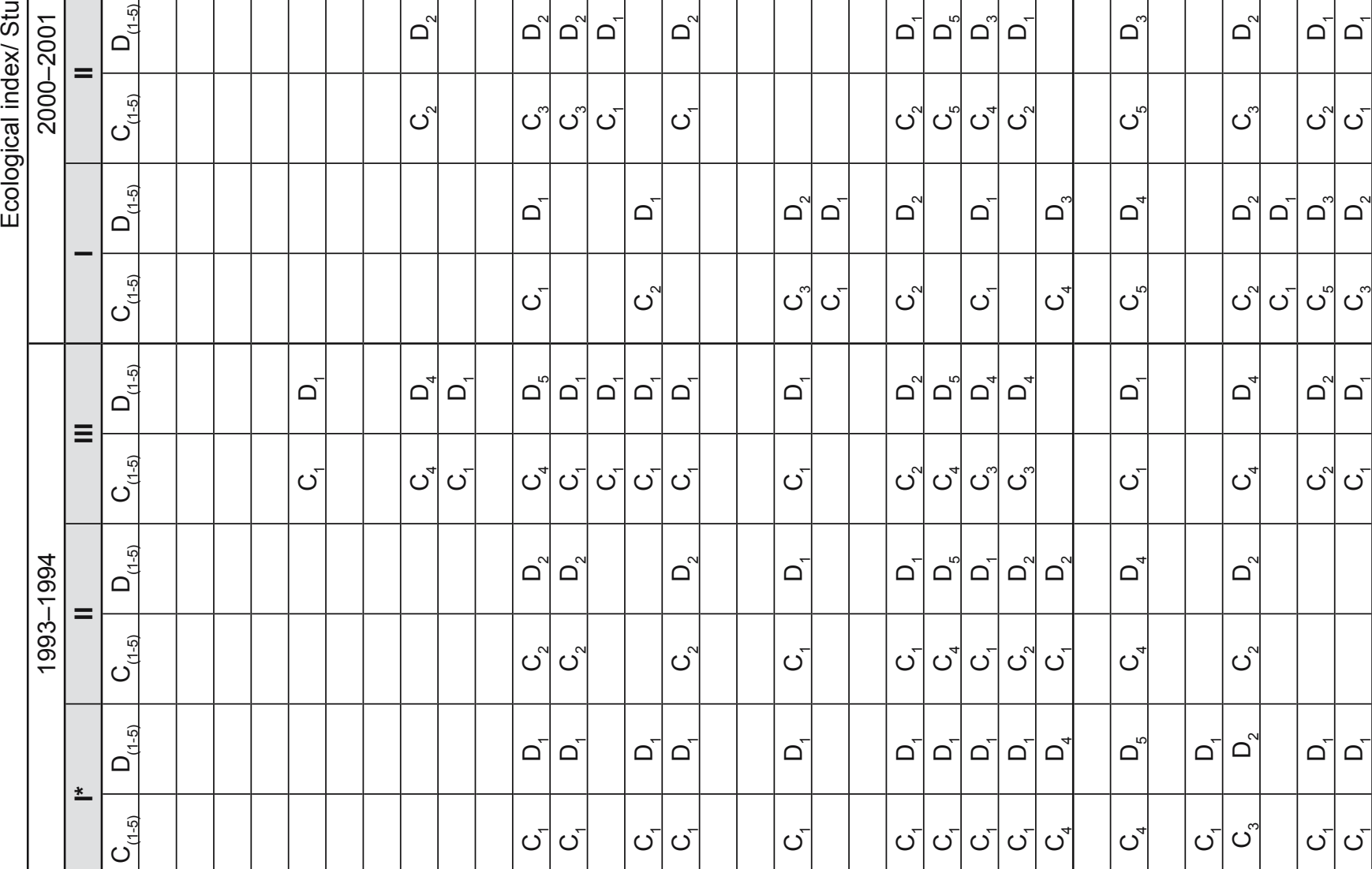

\begin{tabular}{|c|c|c|c|c|c|c|c|c|c|c|c|c|c|c|c|c|c|c|c|c|c|c|c|c|c|c|}
\hline 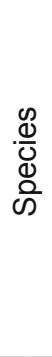 & 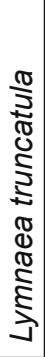 & 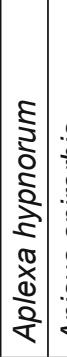 & 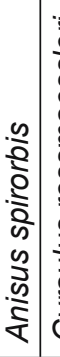 & 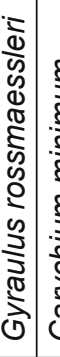 & 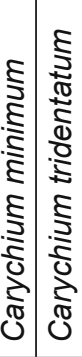 & 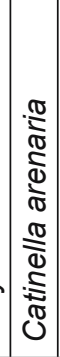 & 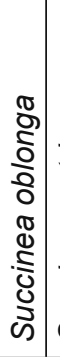 & 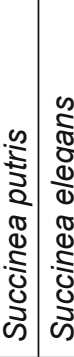 & 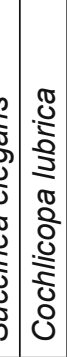 & 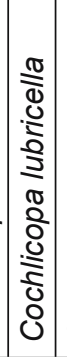 & 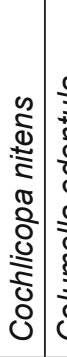 & 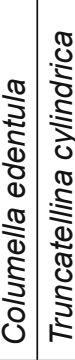 & 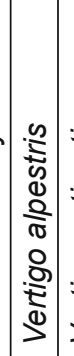 & 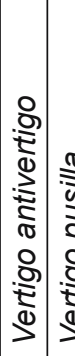 & 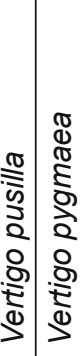 & 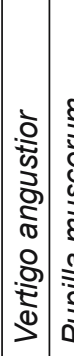 & 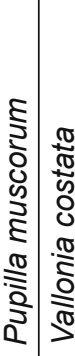 & 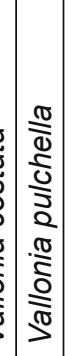 & 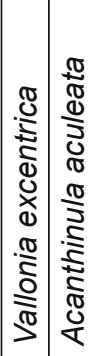 & 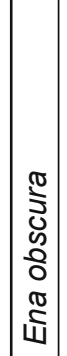 & 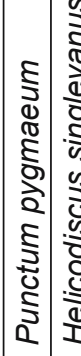 & 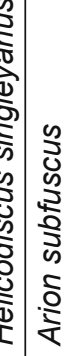 & 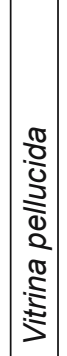 & 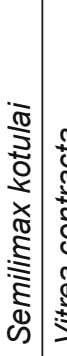 & 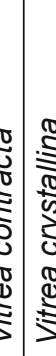 & \\
\hline$\frac{c}{2}$ & $\tau$ & $\sim$ & $m$ & $\nabla$ & \begin{tabular}{l|l}
0 & 0
\end{tabular} & $\wedge$ & $\infty$ & $\infty$ 으 & $=$ & $\cong$ & $\stackrel{m}{\Rightarrow}=$ & $\underset{\sim}{\sim}$ & 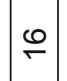 & $\neg:$ & 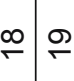 & શิ & $\bar{N} \approx N$ & $\cong$ & $\stackrel{\sim}{\stackrel{N}{N}}$ & 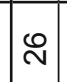 & $\hat{N} \stackrel{\infty}{A}$ & $\begin{array}{l}\stackrel{S}{N} \\
\end{array}$ & প্ల & $\bar{m}$ & $m$ & \\
\hline
\end{tabular}




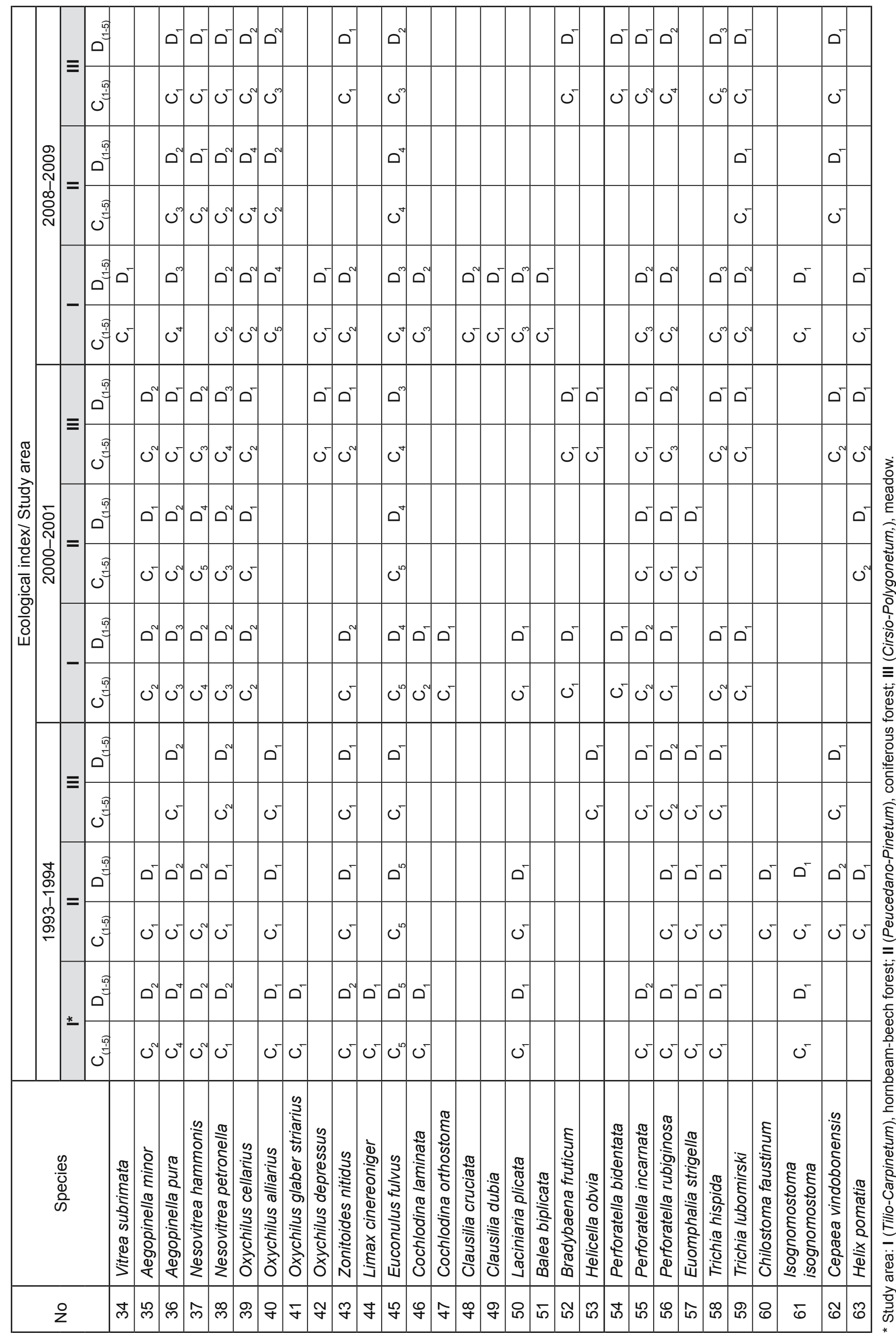


Table 3. Ecological structure of snail communities in the study areas in the 1993-2009 period

\begin{tabular}{|c|c|c|c|c|c|c|c|c|c|c|}
\hline \multirow{2}{*}{ E } & \multirow{2}{*}{ Species } & \multicolumn{3}{|c|}{ 1993-1994 } & \multicolumn{3}{|c|}{ 2000-2001 } & \multicolumn{3}{|c|}{ 2008-2009 } \\
\hline & & $I^{*}$ & II & III & $\mathrm{I}$ & II & III & I & II & III \\
\hline \multirow{13}{*}{1} & Vertigo pusilla (O.F. Müller 1774) & III & III & 1 & IV & - & II & III & - & - \\
\hline & Acanthinula aculeata (O.F.Müller,1774) & VI & III & - & $\mathrm{V}$ & - & - & V & III & - \\
\hline & Ena obscura (O.F.Müller,1774) & - & - & - & - & - & - & II & II & II \\
\hline & Vitrea subrimata (Reinhardt, 1871) & - & - & - & - & - & - & II & - & - \\
\hline & Aegopinella pura (Alder, 1830) & $\mathrm{VI}$ & IV & III & $\mathrm{V}$ & III & II & $\mathrm{V}$ & V & II \\
\hline & Oxychilus depressus (Sterki, 1880) & - & - & - & - & - & II & II & - & - \\
\hline & Limax cinereoniger (Wolf, 1803) & 1 & - & - & - & - & - & - & - & - \\
\hline & Cochlodina laminata (Montagu, 1803) & II & - & - & II & - & - & $\mathrm{V}$ & - & - \\
\hline & Cochlodina orthostoma (Menke, 1828) & - & - & - & II & - & - & - & - & - \\
\hline & Clausilia cruciata (Studer, 1820) & - & - & - & - & - & - & $\mathrm{V}$ & - & - \\
\hline & Perforatella incarnata (O. F. Müller, 1774) & IV & - & II & IV & II & III & $\mathrm{V}$ & - & III \\
\hline & Chilostoma faustinum (Rossmässler, 1835) & - & 1 & & - & - & - & - & - & - \\
\hline & $\begin{array}{l}\text { Isognomostoma isognomostoma } \\
\text { (Schröter, 1784) }\end{array}$ & III & II & - & - & - & - & II & - & - \\
\hline \multirow{7}{*}{2} & Semilimax kotulai (Westerlund, 1883) & - & - & - & 1 & - & - & - & II & - \\
\hline & Vitrea crystallina (O. F. Müller, 1774) & 1 & - & 1 & IV & II & III & $\mathrm{V}$ & III & - \\
\hline & Aegopinella minor (Stabile, 1864) & $\mathrm{V}$ & II & - & IV & II & IV & - & - & - \\
\hline & Oxychilus glaber striarius (Rossmässler, 1835) & $\mathrm{I}$ & - & - & - & - & - & - & - & - \\
\hline & Balea biplicata (Montagu, 1803) & - & - & - & - & - & - & III & - & - \\
\hline & Bradybaena fruticum (O. F. Müller, 1774) & - & - & II & - & - & II & - & - & II \\
\hline & Helix pomatia (Linnaeus, 1758) & - & 1 & - & - & III & III & III & - & - \\
\hline 3 & Perforatella bidentata (Gmelin, 1791) & - & - & - & II & - & - & - & - & IV \\
\hline \multirow{2}{*}{4} & Helicella obvia (Menke, 1828) & - & - & III & - & - & 1 & - & - & - \\
\hline & Cepaea vindobonensis (Férussac, 1821) & - & III & III & - & - & III & - & III & II \\
\hline \multirow{8}{*}{5} & Catinella arenaria (Bouchard-Chantereaux, 1837) & - & - & - & - & - & - & - & - & $\mathrm{I}$ \\
\hline & Truncatellina cylindrica (Férussac, 1807) & 1 & IV & 1 & - & IV & III & - & V & - \\
\hline & Vertigo pygmaea (Draparnaud, 1801) & - & - & - & 1 & - & IV & - & - & - \\
\hline & Pupilla muscorum (Linnaeus, 1758) & 1 & 1 & IV & IV & III & IV & - & $\mathrm{V}$ & $\mathrm{V}$ \\
\hline & Vallonia costata (O. F. Müller, 1774) & II & $\mathrm{VI}$ & $\mathrm{VI}$ & - & VII & $\mathrm{VI}$ & - & $\mathrm{VI}$ & $\mathrm{V}$ \\
\hline & Vallonia pulchella (O. F. Müller, 1774) & 1 & III & $\mathrm{V}$ & 1 & $\mathrm{~V}$ & $\mathrm{VI}$ & - & $\mathrm{V}$ & $\mathrm{VII}$ \\
\hline & Vallonia excentrica (Sterki, 1893) & 1 & IV & $\mathrm{V}$ & - & IV & $\mathrm{V}$ & - & $\mathrm{V}$ & $\mathrm{VI}$ \\
\hline & Euomphalia strigella (Draparnaud, 1801) & II & 1 & 1 & - & 1 & - & - & - & - \\
\hline 6 & Cochlicopa Iubricella (Porro, 1838) & 1 & IV & 1 & I & IV & IV & - & $\mathrm{V}$ & - \\
\hline \multirow{15}{*}{7} & Cochlicopa Iubnica (O.F.Müller,1774) & 1 & IV & $\mathrm{VI}$ & II & $\mathrm{V}$ & $\mathrm{V}$ & II & IV & $\mathrm{VI}$ \\
\hline & Vertigo alpestris (Alder, 1837) & - & - & - & - & - & 1 & - & - & - \\
\hline & Punctum pygmaeum (Draparnaud, 1801) & $\mathrm{VI}$ & $\mathrm{VI}$ & II & $\mathrm{Vl}$ & V & $\mathrm{V}$ & III & IV & - \\
\hline & Helicodiscus singleyanus (Pilsbry, 1889) & - & - & - & - & - & - & II & - & - \\
\hline & Arion subfuscus (Draparnaud, 1805) & III & - & - & - & - & - & - & - & - \\
\hline & Vitrina pellucida (O. F. Müller, 1774) & V & $\mathrm{V}$ & $\mathrm{VI}$ & IV & IV & $\mathrm{V}$ & III & II & $\mathrm{VI}$ \\
\hline & Vitrea contracta (Westerlund, 1871) & 1 & - & IV & $\mathrm{V}$ & III & - & $\mathrm{V}$ & - & - \\
\hline & Nesovitrea hammonis (Ström, 1765) & IV & IV & - & $\mathrm{V}$ & $\mathrm{VI}$ & $\mathrm{V}$ & - & III & II \\
\hline & Oxychilus alliarius (Miller, 1822) & III & III & III & - & - & - & $\mathrm{VI}$ & IV & $\mathrm{V}$ \\
\hline & Oxychilus cellarius (O. F. Müller, 1774) & - & - & - & IV & 1 & IV & IV & $\mathrm{V}$ & V \\
\hline & Euconulus fulvus (O. F. Müller, 1774) & $\mathrm{VI}$ & VI & III & $\mathrm{VI}$ & $\mathrm{VI}$ & $\mathrm{V}$ & $\mathrm{V}$ & $\mathrm{VI}$ & IV \\
\hline & Clausilia dubia (Draparnaud, 1805) & - & - & - & - & - & - & III & - & - \\
\hline & Laciniaria plichta (Draparnaud, 1801) & III & III & - & II & - & - & $\mathrm{V}$ & - & - \\
\hline & Trichia hispida (Linnaeus, 1758) & III & II & II & II & - & IV & $\mathrm{V}$ & - & $\mathrm{VI}$ \\
\hline & Trichia Lubomirski (Ślósarski, 1881) & - & - & - & II & - & I & IV & II & II \\
\hline
\end{tabular}


Table 3. Ecological structure of snail communities in the study areas in the 1993-2009 period - cont.

\begin{tabular}{|c|c|c|c|c|c|c|c|c|c|c|}
\hline \multirow{2}{*}{ E } & \multirow{2}{*}{ Species } & \multicolumn{3}{|c|}{ 1993-1994 } & \multicolumn{3}{|c|}{$2000-2001$} & \multicolumn{3}{|c|}{$2008-2009$} \\
\hline & & $I^{*}$ & II & III & $\mathbf{I}$ & II & III & 1 & II & III \\
\hline \multirow{5}{*}{8} & Carychium tridentatum (Risso, 1826) & - & - & - & - & - & I & - & - & V \\
\hline & Succinea oblonga (Draparnaud, 1801 & - & - & V & - & IV & V & - & IV & VII \\
\hline & Columella edentula (Draparnaud, 1805) & IV & - & II & III & - & III & IV & - & $\mathrm{VI}$ \\
\hline & Vertigo angustior (Jeffreys, 1830) & - & - & - & - & - & - & - & - & IV \\
\hline & Nesovitrea petronella (L. Pfeiffer, 1853) & IV & II & IV & IV & V & $\mathrm{V}$ & IV & IV & IV \\
\hline \multirow{7}{*}{9} & Carychium minimum (O.F.Müller,1774) & - & - & 1 & - & - & 1 & - & - & - \\
\hline & Succinea putris (Linnaeus, 1758) & - & - & II & - & - & III & - & - & III \\
\hline & Succinea elegans (Risso, 1826) & - & - & - & - & - & - & - & - & III \\
\hline & Cochlicopa nitens (Gallenstein, 1852) & - & - & 1 & - & III & III & - & - & IV \\
\hline & Vertigo antivertigo (Draparnaud, 1801) & - & - & - & - & - & - & - & - & II \\
\hline & Zonitoides nitidus (O. F. Müller, 1774) & IV & 1 & III & IV & - & IV & IV & - & IV \\
\hline & Perforatella rubiginosa (A. Schmidt, 1853) & III & II & $\|$ & 1 & 1 & IV & IV & - & $\mathrm{V}$ \\
\hline \multirow{6}{*}{10} & Aplexa hypnorum (Linnaeus, 1758) & - & - & - & - & - & II & - & - & II \\
\hline & Lymnaea truncatula (O.F.Müller,1774) & - & - & - & - & - & - & - & - & II \\
\hline & Anisus spirorbis (Linnaeus, 1758) & - & - & - & - & - & III & - & - & - \\
\hline & Gyraulus rossmaessleri (Auerswald, 1851) & - & - & - & - & - & - & - & - & IV \\
\hline & $N_{t}$ & 31 & 26 & 29 & 28 & 23 & 37 & 29 & 22 & 33 \\
\hline & $\mathrm{N}_{\mathrm{s}}$ & 3921 & 2879 & 2684 & 2806 & 3122 & 3684 & 2636 & 2700 & 7816 \\
\hline
\end{tabular}

* Study area: I (Tilio-Carpinetum), hornbeam-beech forest; II (Peucedano-Pinetum), coniferous forest; III (Cirsio-Polygonetum,), meadow.

The classes of the number species were introduced in the logarithmic scale I-VIII:

I - 1-3, II - 4-9, III - 10-31, IV - 32-99, V - 100-316, VI - 317-999, VII - 1000-3162, VIII - 3163-9999.

$\mathrm{N}_{\mathrm{t}}$ - number of species, $\mathrm{N}_{\mathrm{s}}-$ number of individuals

In the next years, the number of species at site I slightly decreased. I. isognomostoma and two nude species Arion subfuscus and Limax cinereoniger, the body of which is more sensitive to dust pollution, were not noted. Mezophilous and forest species constituted $68 \%$ including $17 \%$ of forest species. The numbers of abundant hygrophilous and hydrophilous species remained at the same level. Species of dry meadows were scarce. Profuseness of the Pupilla muscorum population was probably due to shallow rock-mantle loam in some microhabitats which keeps humidity and is particularly preferred by this species. In the years 2000-2001 the number of forest species increased to 34\%. In 2008-2009 mezophilous and forest species composed together $93 \%$ of all snail species. The percentage of hygrophilous and hydrophilous species remained at the same level. No species typical of dry meadows (E5) were noted which could be explained by a lower carbonate dust deposition in soil.

At site II (coniferous forest) soils belonged to autogenic rusty podzolic soils formed from deluvial sands overlaying fluvioglacial sands. These sands surrounded the Malik Hill with a ring and were of landslide/sedimentary character. The rusty podzolic soil under study contained up to $6 \%$ of fine particles and $7 \%$ of dust fraction in the bottom part of the profile and $10 \%$ of fine fraction and $16 \%$ of dust fraction in the top part of the profile. Many-years' cement dust deposition strongly alkalized the habitat. In 1992 the $\mathrm{pH}_{\mathrm{KCl}}$ values ranged from 5.90 to 6.74 , while carbonate contents were up to $3.24 \%$. In $2009 \mathrm{pH}$ ranged from 5.22 to 6.63 while $\mathrm{CaCO}_{3}$ content did not exceed $2.54 \%$. Saturation of sorption complex was unnaturally high for this soil type exceeding $<83 \%$ in 1992 and $<81 \%$ in 2009 (Tab. 1). The sum of bases S1, with the dominating calcium, ranged from $3.10 \mathrm{cmol}(+) / \mathrm{kg}$ in parent rock to $56.91 \mathrm{cmol}(+) / \mathrm{kg}$ in Ol horizon (1992). In $2009 \mathrm{~S} 1 \mathrm{in}$ the litter was slightly lower and amounted to $51.21 \mathrm{cmol}(+) / \mathrm{kg}$. The AEes horizon contained $0.92 \%$ of organic carbon (1992) and $0.87 \%$ Corg in 2009 (see Tab.1). Deposition of calcium of anthropogenic origin in these naturally acidic soils improved ecological conditions for snails inhabiting them, influencing their density and species compositions.

At site II in 1993-1994, 26 species were recorded which significantly exceeded naturally poor in species coniferous forest habitats. E. fulvus $\mathrm{C}_{5} \mathrm{D}_{5}$, V. costata $\mathrm{C}_{4} \mathrm{D}_{5}$ and P. pygmaeum $\mathrm{C}_{4} \mathrm{D}_{4}$ were indicator species. In 2000-2001, 23 snail species were documented (Tab. 3). The xerophilous and calciphilous species Vallonia costata $\mathrm{C}_{5} \mathrm{D}_{5}$, and two mezophilous species $N$. hammonis $\mathrm{C}_{5} \mathrm{D}_{4}$ and $E$. fulvus $\mathrm{C}_{5} \mathrm{D}_{4}$. conspicuously dominated. The 2008-2009 survey recorded 22 species with the same number of indicator species. V. costata $\mathrm{C}_{5} \mathrm{D}_{5}$, O. cellarius $\mathrm{C}_{4} \mathrm{D}_{4}$ and E. fulvus $\mathrm{C}_{4} \mathrm{D}_{4}$ dominated. Overrepresentation of several species indicates a strong disturbance in this habitat. High rank of the strongly xerophilous species of open-land habitats $V$. costata in this community suggests difficult humidity conditions and light forest floor. The number of companion species decreased in successive years which could be linked with the lowered alkalization and more difficult humidity conditions which slowed down regeneration of the coniferous forest habitat. 

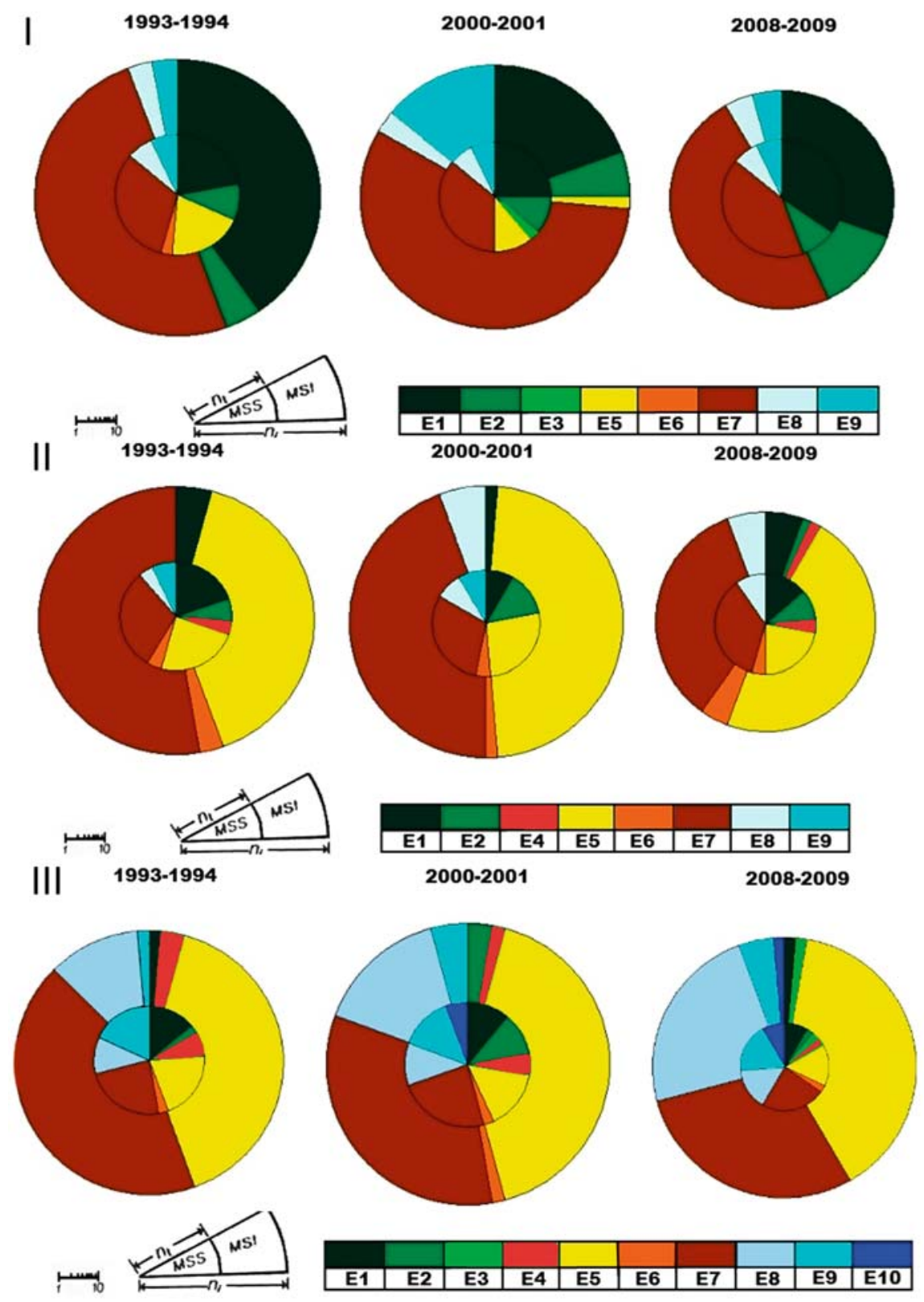

Fig. 2. Malacospectra of species

I* area: I (Tilio-Carpinetum), hornbeam-beech forest; II (Peucedano-Pinetum), coniferous forest; III (Cirsio-Polygonetum,), meadow. MSS - malacospectra of species, MSI - malacospectra of individuals

E1-E10: 1 - forest species, 2 - shadowy partly species, 3 - moist forests species, 4 - xero-thermophilous species, 5 - open-country species, 6 - mesophilous species, 7 - medium-dry-county species, 8 - medium-humid species, 9 - hydrophilous species, 10 - water species, dryling periodically

The proportion of forest and mezophilous species amounted to $65 \%$, including $19 \%$ of forest species. The proportion of dry meadow species (E5) was high reaching 27\% (Fig. 2). The species preferring higher humidity occurred sporadically. In the 2000-2001 survey, the percentage of forest and mezophilous species declined to $61 \%$ of which $9 \%$ belonged to forest species. Dry meadow species still comprised $26 \%$ of all species and overrepresentation of $V$. costata. still persisted. In 2008-2009, the proportion of mezophilous and forest increased to $73 \%$, including $14 \%$ of forest species. A total of $27 \%$ of all species belonged to dry meadow species which were very abundantly represented. Hydrophilous species were absent.

At site III (meadow) soil was defined as proper gley soil. It was located on a wide depression at the foot of the Malik Hill. This soil was formed from loamy aluvias of the Bobrzyczka River. Surface horizons were composed of light loams containing $31 \%$ of fine fraction whereas bottom horizons comprised medium loams slightly enriched in dust fraction $(17 \%)$. The soil was humid, periodically flooded (particularly in spring). Thus, all soil processes occurred under constant oxygen deficit which aggravated with depth. This was evidenced by numerous blue and greenish steely $(2,5 \mathrm{Y}$ $7 / 3,2,5 \mathrm{Y} 6 / 2$ ) stains in mineral (Gr, CGr) horizons of the soil profile. Chemical properties of gley soil were also strongly modified by limestone dust deposition. The $\mathrm{pH}_{\mathrm{KCl}}$ value decreased with depth from 6.64 to 5.35 as measured in 1992 and from 6.43 to 5.12 in 2009 . These were the lowest $\mathrm{pH}_{\mathrm{KCl}}$ values of all the samples studied. Carbonates occurred in the 
analyzed profile to a depth of $15 \mathrm{~cm}$ (max. from $1.2 \% \mathrm{CaCO}_{3}$ in 2009 to $1.35 \%$ in 1992). Surface soil layer is associated with circadian activity of snails and their biology. It is also the place of snail hibernation in winter. Base saturation of sorption complex was $57.12-82.96 \%$ in 1992 and $52.2-83.44 \%$ in 2009 , while the sum of exchangeable bases S1 ranged from 14.36 to $44.21 \mathrm{cmol}(+) / \mathrm{kg}$ in 1992 and from 10.16 to $41.05 \mathrm{cmol}(+) / \mathrm{kg}$ in 2009. The humic horizon with marked depth and organic matter content additionally supported snail development. At site III, the 1993-1994 survey recorded 29 species with 4 indicator species: V. costata $\mathrm{C}_{4} \mathrm{D}_{5}$, Cochlicopa lubrica $\mathrm{C}_{4} \mathrm{D}_{5}$, Succinea oblonga $\mathrm{C}_{4} \mathrm{D}_{4}$ and $V$. pellucida $\mathrm{C}_{4} \mathrm{D}_{4}$. In the subsequent study period of 2000-2001, 37 species were identified and V. costata $\mathrm{C}_{5} \mathrm{D}_{4}$ and V. pulchella $\mathrm{C}_{5} \mathrm{D}_{4}$ were indicator species. In 2008-2009, the number of species amounted to 37 and the number of indicator species increased to five: $S$. oblonga $C_{5} D_{4}$, C. lubrica $C_{5} D_{4}, V$. pulchella $C_{5} D_{4}$,Vexcentrica $C_{5} D_{4}$ and Vitrina pellucida $C_{5} D_{4}$. Availability of calcium ions and good humidity at site III created favorable conditions for malacofauna development which was reflected by the dominance of meadow species. At this site, three meadow species Succinea oblonga, $V$. costata and C. lubrica. were the most abundant throughout the study period from 1993 to 2001. Species of open-land habitats (E4, E5) made $27 \%$ of all species, including the xerothermic species $V$. costata which occurred in masses. Species of shady habitats were sporadic and their percentage amounted to 17 . Species of wet habitats also constituted $17 \%$ of snail species, and individual species were scarcely represented. Snails of open dry habitats were represented by $28 \%$ species, including $V$. costata the massive occurrence of which was recorded again. In these years, mezophilous and forest species comprised $49 \%$ of all species but species preferring shady habitats (E1 and E2) were scarce and of accessory character. Species of humid habitats and wetlands (E9 and E10) were also scanty and constituted $19 \%$ of the whole assemblage. On the other hand, euryecological (E7) species were abundant which indicates anthropogenic character of the meadow. Xerophilous open-land habitats harbored only $15 \%$ of species with the calciphilous xerophilous species $V$. costata still abundantly represented. In the next years, a new earlier unreported species appeared in the meadow, i.e. Perforatella bidentata $\mathrm{C}_{1} \mathrm{D}_{1}$ preferring wet woodlands (E3). The proportion of species of wet and aquatic habitats (E9 and E10) was higher reaching $26 \%$, i.e. $9 \%$ more than in previous years, they were also more abundant. Mezophilous and forest habitats harbored 50\% of all species, of which hygrophilous (E8) and euryecological species (E7) were numerous. The hygrophilous species Columella edentula typical of wetlands was also profusely represented. In 2009, the rare species Vertigo angustior was discovered on the meadow which was important for assemblage formation and inhabited lowland bogs and wetlands. This very sensitive, hygrophilous and calciphilous species lives only in natural habitats. Accessory species included snails of wet meadows, i.e. distinctly calciphilous and hydrophilous rare species Cochlicopa nitens and hydrophilous species Perforatella rubiginosa.

Malacofauna studies conducted on the Malik Hill in the years 1993-2009 documented the presence of 63 species of snails belonging to 17 families. In the period 1993-1994 density of snails was estimated at 980 snails $/ 1 \mathrm{~m}^{2}$ at site I, 720 snails $/ 1 \mathrm{~m}^{2}$ at site II and 671 snails $/ 1 \mathrm{~m}^{2}$ at site III. In
2000-2001, snail densities remained at a similar level amounting to 702 snails $/ 1 \mathrm{~m}^{2}$ (site I), 780 snails $/ 1 \mathrm{~m}^{2}$ (site II) and 921 snails $/ 1 \mathrm{~m}^{2}$ (site III). In the 2008-2009 survey, the respective densities were 659 snails $/ 1 \mathrm{~m}^{2}$ (site I), 675 snails $/ 1 \mathrm{~m}^{2}$ (site II) and 1954 snails $/ 1 \mathrm{~m}^{2}$ (site III). In reference to the studies by Hawkins et al. (1997), snail densities were at the medium level at site I and II and were high at site III. Dynamics of malacofauna changes at three different sites exposed to alkaline dust pollution was characterized by an increased number of dominating species and numerous accessory species.

The studies carried out at the same sites in 2009 confirmed that the decreased cement dust emission resulted only in a slight lowering (by from $2 \%$ to $10 \%$ ) of the studied most alkalinization-dependent soil characteristics: $\mathrm{pH}$, carbonate content and soil base saturation. The greatest differences were observed in successive years in surface soil horizons, being the least significant in the parent rock. The rusty podzolic soil reacted in the strongest way to the reduced alkaline deposition (site II). Soil and snail studies in the period of 1994-2009 evidenced that the changes in soil conditions at three study sites on the Malik Hill influenced development of malacocenoses.

\section{Discussion}

The Świętokrzyskie Mountains belong to the most thoroughly studied and the richest regions of Poland in terms of the number of malacofauna species (Barga-Więcławska 1997, 2005, Riedel 1988). In the 1960s, comprehensive studies conducted by Piechocki (1981) on natural habitats of the Chęciński Region identified 13 snail species on the Malik Hill and in its surroundings. Small number of species was due to a non-standard method used at that time. In 2008-2009, 50 species from 17 families, including 46 terrestrial species and 4 aquatic snail species were recorded at study sites.

It was evidenced that, compared to the beginning of research, species composition changed and the number of species increased by 11 . The number of families also increased and the number of terrestrial species characteristic of wet and aquatic habitats rose by 6 . The number of snail species increased in this area because of deposition of exhaust dust in soils causing alkalization of their surface, i.e. the most biologically active soil horizons (Kowalkowski and Świercz 1993, Świercz 2005, 2006). Cement and lime dust deposit on plants and litter create new environment for snail growth, e.g. due to an increase in $\mathrm{pH}$ values and disturbance of humidity of habitats which was confirmed by gradation of $V$. costata. Species composition and numbers of snails depended also on feeding conditions and nutrient availability at study sites.

Investigations of lichens in the surroundings of Nowiny village conducted by Łubek (2010) demonstrated a significant reduction of biodiversity. It was established that deposition of dust emitted from the cement plant caused unification of physicochemical properties of bark of different tree species and caused that biota of calciphilous epiphytes resistant to drying became uniform. Epiphytes occurred in masses completely covering tree trunks. Drastically changed feeding conditions for Clausiliidae explain why their population was becoming extinct in the years 1993-2001. Appearance of an abundant population of the stenotopic species Clausilia cruciata and more tolerant Cochlodina laminata, Balea 
biplicata and Clausilia dubia, and also Perforatella incarnata belonging to the family Helicidae feeding on trees indicates that dust deposition was declining which improved ecological conditions at site III.

At study sites on the Malik Hill, the changes in natural soil acidity, together with the increase in their alkalization and $\mathrm{CaCO}_{3}$ accumulation led to diminution of organic matter content which had to influence species feeding on algae and detritus. Similar conclusions can be found in the report of Simpson (2012). Snails, depending on their structure and ecological requirements, live in soil, litter (mostly $\mathrm{Ol}$, Ofh horizons), on rotting wood and on some plant species. Snails are also dependent on vegetation, i.e. different plant communities harbor characteristic snail assemblages (Abele 2010, Gosteli 1996). Snail studies conducted in different forest associations (Addison and Barber 1997, Niemela 1997) indicated that terrestrial snails were strongly dependent on litter composition and were the most abundant in multispecies deciduous forests. According to Götmark et al. (2008), studies carried out in 25 forest reserves indicated that $\mathrm{pH}$ of the litter was the most important factor decisive for the composition and abundance of snails. The malacofauna of the Malik Hill inhabits three different plant communities (hornbeam-beech woods, coniferous forest and meadow) growing on distinct soil types: rendzinas developed on Devonian dolomites, sandy rusty podzolic soils and gley soils which have been exposed to anthropogenic pressure for many years. It has been proven that snails strongly depend on geological substratum (Barga-Więcławska 1997). Succession of snails on the carbonate substratum, typical of Chęciny District, was shown to be very dynamic and preceded the succession on silica substratum. Cement and lime dust deposition in soils influences their physicochemical properties and malacofauna composition. It should be remembered that primary dust deposition and secondary from roads overlap. The dust emitted from the Dyckerhoff Cement Plant was composed mostly of $\mathrm{CaO}$ (49.5-66.6\%), $\mathrm{SiO}_{2}(14,0-15.4 \%), \mathrm{Al}_{2} \mathrm{O}_{3}$ č(3.9-4.9\%), $\mathrm{K}_{2} \mathrm{O}$ (3.8-4.1\%) and others. It was confirmed by studies of Świercz (1997, 2006) and Mandre and Lukjanova (2011). According to the particle size distribution studies, they contained $62 \%$ of fine fraction which increased the scope of their impact (Kumari and Pandey 2011, Świercz 1997, Tervahattu et al. 2001).

Due to anatomical structure, snails require calcium ions $\mathrm{Ca}^{+2}$ which they take up from water. The significance of calcium ion availability for snails was addressed by Horsak et al. (2007), Horsak and Hajek (2003), Martin and Sommer (2004), Juričkova et al. (2008). Snails are able to store large amounts of calcium (Fourniae and Chetail 1982), however, its excess is toxic. They can regulate the amount of calcium by intracellular and extracellular precipitation and by elimination (Fourniae and Chetail 1982). Limestone karsted substratum at site I was beneficial for snails because of the abundance of refuges. Natural carbonate compounds contained in rendzinas were complemented by deposition of anthropogenic origin which improved living conditions for malacocenoses. Site I was characterized by beneficial humidity and abundance of nutrients (humus of kalci-mull type). Calcium dominated among exchangeable cations. The effect of alkaline emission was less visible on naturally alkaline rendzinas but the presence of $\mathrm{CaCO}_{3}$ in organic horizons indicates its anthropogenic origin (Ade-Ademilua, Obalola 2008). In contrast, site II was influenced by alkalization in a strongest way. Podzolic soils naturally develop from carbonate-free sand formations poor in nutrients. The rusty podzolic soil at site II lost its primary properties due to exposure to cement and lime industry influences (see Tab. 1). Its profile structure and particle size distribution remained unchanged which was proven in earlier reports (Kowalkowski and Świercz 1993, Degórski 1998, Jaworska et al. 2010). However, both organic and mineral horizons were enriched in calcium compounds which radically changed their $\mathrm{pH}$ and saturation of sorption complex. Paradoxically, the strong transformation of chemical properties of this soil contributed to its increased attractiveness to malacofauna. Despite elevation of eutrophication of anthropogenic origin, the coniferous forest habitat was less abundant in assimilable matter for snails (leaf litter from coniferous and deciduous trees), it was characterized by low humidity and lack of natural refuges. Conditions of the coniferous forest ecosystem were the most difficult for snail development.

However, humidity remains the main factor limiting development of snails (Alexandrowicz and Aleksandrowicz 2011). Conspicuous quick reaction of malacofauna to changes in ground water level was documented by the studies of Barga-Więcławska (2009) in the Kampinos Forest. Good humidity in river valleys increased resistance of snails to anthropogenic pressure (Baba et al. 1992). The study sites on the Malik Hill differed in humidity level, with site III having the most beneficial conditions. This habitat was humid, periodically flooded by the river. Gley soil showed beneficial $\mathrm{pH}$ values $\mathrm{pH}_{\mathrm{KCl}}>5.12$ and slight pollution with anthropogenic carbonates in surface horizons (to $15 \mathrm{~cm}$ ). Organic horizon, most intensely penetrated by snails, was composed of fermenting herbaceous vegetation and contained about $37 \%$ Corg.

In the years 1993 - 1994, at site I and II, no auxiliary species were distinguished, several species dominated while accessory species were numerous. After seven years had passed, the disturbance in domination structure of snails deepened. The rank of dominant forest species declined. At sites I and II, gradation of calciphilous species of xerothermic meadows Vallonia costata increased. Clausiliidae living in the litter and on trees and other rare species withdrew from site I (hornbeam-beech woods). In the years 2008-2009, humidity conditions in habitats improved which allowed snail communities to regain features of normal constancy and dominance distribution. The changes in soil conditions at study sites connected with changes in $\mathrm{pH}$ values, active carbonate contents and hydrolytic acidity particularly in the surface soil horizons and improvement of conditions on trees and in leaf litter were decisive for species composition and ecological structure of terresrtial snails. In the study years, chemical characteristics of soils only slightly changed in spite of abrupt lowering of cement and lime dust deposition in soils. The changes in malacocenoses in consecutive years were apparent and had dynamic character. Currently, only slight alkalization of the environment with sufficient humidity of soils decidedly supports regeneration of habitats and development of snails.

\section{Conclusions}

Soil studies demonstrated that deposition of alkaline dust had the strongest impact on properties of rusty podzolic soil (site II) causing its completed deacidification. Decreasing dust 
emission to the atmosphere was accompanied by the following tendencies in soil horizons: lowering of $\mathrm{pH}$ value mostly in organic and humic horizon, slight lowering of exchangeable cation contents in sorption complex in all studied soils, lowering of carbonate content in all studied soils, slight increase in hydrolytic acidity.

Despite the decrease in cement and lime dust deposition, chemical properties of soils under study were still dependent of a long-term alkalization which was confirmed by the stable population of malacofauna.

Malacofauna studies demonstrated an increase in total number of snail species from 39 to 50, including 4 aquatic species. The number of species at site I (hornbeam-beech woods) remained at the same level throughout the whole study period. At site II (coniferous forest), the number of species showed a decreasing tendency while the number of species at site III (meadow) presented an increasing trend. Snail density at sites I and II (hornbeam-beech woods and coniferous forest) persisted at the same level whereas at site III (meadow) it increased thrice by 2009. After a 30-year period of incessant alkalization, in 1993-1994, at sites I and II the domination structure of snail communities was distinctly disturbed which was manifested by the absence of auxiliary species. In subsequent years, the number of species characterized by greater constancy increased while the number of rare species declined. In 2008-2009, the number of species with high constancy and the number of rare species distinctly rose at sites I and III (hornbeam-beech woods and meadow) and remained at the same level at site II (coniferous forest).

The impact of alkaline dust emitted from the cement plant was the greatest at site II which was confirmed by a large number of species and high snail density differing from normal values in coniferous forest habitats. The most beneficial habitat conditions for malacofauna development were observed at site I - hornbeam-beech woods on proper rendzina, and at site III - meadow on gley soil. Species composition and structure of malacocenose at site I (hornbeam-beech woods) suggest a natural character of this habitat and its high resistance to alkaline anthropogenic pressure.

The studies of malacocenoses in reference to physicochemical characteristics of habitats justify the use of snails as a sensitive bioindicator of environmental changes.

\section{References}

Abele, S.E. (2010). Gastropod diversity in the boreal mixedwood forest of northern Alberta - variation among forest types and response to partial harvesting, M.S. Thesis. University of Alberta, Edmonton, Alberta 2010.

Ade-Ademilua, O.E. \& Obalola, D.A. (2008). The effect of cement dust pollution on Celosia Argentea (Lagos Spinach) Plant, Journal of Environmental Science and Technology, 1 (2), pp. 47-55.

Addo, M., Darko, E., Gordon, C. \& Nyarko, B.J.B. (2013). Contamination of soils and loss of productivity of (Vigna unguiculata L.) caused by cement dust pollution. Research Journal of Chemistry and Environment, 3,1, pp. 272-282.

Addison, J.A. \&. Barber, K.N.(1997). Response of soil invertebrates to clearcutting and partial cutting in a boreal mixedwood forest in Northern Ontario, Natural Resources Canada, Canadian Forest Service, Great Lakes Forestry Centre. Information Report GLC-X-1, Canada 1997.
Alexandrowicz, S.W. (1987). Malakofauna late Holocene Vistulian and central part of the Malopolska Upland, Prace Naukowe Uniwersytetu Śląskiego, 712, Katowice 1987. (in Polish)

Alexandrowicz, S.W. \& Alexandrowicz, W.P. (2011). Malacological analysis. Test methods and interpretation, Rozprawy Wydziału Przyrodniczego, Kraków, t. 3. (in Polish)

Baba, K. \& Podani, J. (1992). A multivariate analysis of snail distribution in the Bükk Mountains, Hungary, Proceedings of the Ninth International Malacofauna Congress, pp. 35-90.

Baba, K., Sárkány \& Kiss, A. (2008). Terrestrial snail fauna in the Somes/Szamos river Valley from the spring region to the river Tisza, Tiscia Monograph, pp. 279-296.

Barga-Więcławska, J. (1997). Succession snails in landfills Świętokrzyskie region, Wyższa Szkoła Pedagogiczna, Kielce 1997. (in Polish)

Barga-Więcławska, J. (2005). Snails sensitive biomarkers environmental acidification in the Lysogóry, Biblioteka Monitoringu Środowiska, Poznań, pp. 335-346. (in Polish)

Barga-Więcławska, J. (2009). Southern strip of Kampinos Park moors malakofauna as an indicator of ecological conditions in its habitats, Kampinoski Park Narodowy, Izabelin, pp. 387-396. (in Polish)

Beeby, A. \& Richmond, L. (2004). Do the soft tissues of helix aspersa serve as a quantitative sentinel of predicted free lead concentrations in solis? Applied Soli Ecology, 22, 2, pp. 159-165.

Brożek, S. \& Zwydak, M. (2003). Polish Atlas forest soils. Centrum Informacyjne Lasów Państwowych 2003. (in Polish)

Chétail, M. \& Krampitz, G. (1982). Calcium and skeletal structures in mollusks: concluding remarks. Proceedings of the 7 th. International Malacofauna Congress Malacologia, 22, (1-2), pp. 337-339.

Degórski, M. (1998). Physico-chemical differentiation of the soil properties of pine and Mied pine forest habitats along a transect of climate and a "Silesian" transect. Dokumentacja Geograficzna, 13, pp. 41-53. (in Polish)

Derome, J., Kukkola, M. \& Malkonen, E. (1986). Forest liming on mineral soils, National Swedish Environmental Protection Board. Report 3084, Solna 1986.

Fourniaé, J. \& Chétail, M. (1982). Accumulation calcique au niveau cellulaire chez les Mollusques, Malacologia, 22, pp. 265-284.

Götmark, F., von Proschwitz, T. \& Franc, N. (2008). Are small sedentary species affected by habitat fragmentation? Local vs. landscape factors predicting species richness and composition of land mollusks in Swedish conservation forests, Journal of Biogeography, 35,(6), pp. 1062-1076.

Gosteli, M. (1996). Diversities of snail fauna and ecological relationships between snail communities and vegetation in dry habitats of the northern Swiss Jura (Gastropoda, Prosobranchia, Pulmonata) Malakologische Abhandlungen Staatliches Museum für Tierkunde Dresden, 18, (10), pp.102-128.

Hawkins, J.W., Lankester, M.W., Lautenschlager, R.A. \& Bell, F.W. (1997). Effects of alternative conifer release treatments on terrestrial gastropods in northwestern Ontario, Forestry Chronicle, 73, pp. 91-98.

Horsak, M. \& Hajek, M. (2003). Composition and species richness of molluscan communities in relation to vegetation and water chemistry in the western Carpathian spring fens: the poovrich gradient, Journal of Molluscan studies, 69, pp. 349-357.

Horsak, M., Hajek, M., Tichy, L. \& Juričova, L. (2007). Plant indicator values as a tool for land mollusc autecology assessment, Acta Oecologica, 3, pp. 161-171.

Hylander, K. (2011). The response of land snails assemblages below aspens to forest fire and clear-cutting in Fennoscandian boreal forests, Forest Ecology and Management, 261, pp. 1811-1819. 
Iqbal, M.Z. \& Shafig, M. (2001). Periodical effect of cement dust pollution on the growth of some plant species, Turkish Journal of Botany, pp. 19-24.

Jaworska, H., Dąbrowska-Naskręt, H. \& Sawilska, K. (2010). The influence of cement dust on some properties of soils and the state of pine stands from the "Lafarge" factory in Bielawy, Proceedings of ECopole, 4(1), pp 141-146. (in Polish)

Juričkova, L., Horsak, M., Cameron R., Hylander, M., Mikovcova, A., Hlavac, J.C. \& Rohovec, J. (2008). Land snail distribution patterns within a site: The role of different calcium sources, European Journal of Soil Biology, 44, pp. 172-179.

Kappes, H. (2005). Influence of coarse woody debris on the gastropod community of a managed calcareous beech forest in Western Europe, Journal of Molluscan Studies, 71(2), pp. 85-91.

Kappes, H. (2006). Relations between forest management and slug assemblages (Gastropoda) of deciduous regrowth forests, Forest Ecology and Management, 237, pp. 450-457.

Kappes, H., Toop, W., Zach, P. \& Kufan, J. (2006). Coarse woody debris, soil properties and snails (Molusca: Gastropoda) in European primeval forests of different environmental conditions, European Journal of Soil Biology, 42(3), pp. 139-146.

Kowalkowski, A. \& Świercz, A. (1993). Parent rocks lithogenic and autogenic soils Malik Hill in the Holy Cross Mountains, Rocznik Świętokrzyski, 14, pp. 91-106. (in Polish)

Kozłowski, R. (2013). The functioning of the Polish geoecosystems selected varied in terms of human pressure on the example of low mountains and foothills, Landform Analysis, 23, 2013. (in Polish)

Kumari, A. \& Pandey, D.D. (2011). Response of maize to cement dust pollution, World Journal of Science and Technology, 1(5), pp. $150-155$.

Ložek, V. (1964). Quartärmollusken der Tschechoslowakei-Rozpravy Ústředního ústavu geologického, Praha, pp. 31-374, Praga 1964. (in Czech)

Łubek, A. (2010). Lichenes [In:] A Monograph of the Chęcińsko-Kielecki Landscape Park, UJK, Kielce, pp. 191-197. (in Polish)

Mandre, M. \& Lukjanova, A. (2011). Biochemical and structural characteristics of scots pine in alkaline environment, Estonian Journal of Ecology, 60,(4), pp. 264-283.

Martin, K. \& Sommer, M. (2004). Relationships between land snail assemblage patterns and soil properties in temperate-humid forest ecosystems, Journal of Biogeography, 3 (4), pp. 531-542.
Niemelä, J. (1997). Invertebrates and boreal forest management, Conservation Biology 11, pp. 601-610.

Oekland, F. (1930). Quantitative Untersuchungen der landschneckenfauna, Norvegens. (in German)

Zeitschrift für Morphologie und Ökologie der Tiere, Berlin, 16 (3-4), pp. 748-804.

Ondina, P., Hermida, J., Outeri, A. \& Mato, S. (2004). Relationships between terrestial gastropod distribution and soil properties in Galicia (NW Spain), Applied Soil Ecology, 26, (1), pp. 1-9.

Ostrowska, A., Gawliński, S. \& Szczubiałka, Z. (1991). ). Methods of analysis and assessment of soil properties and plant, first ed., Institute of Environmental Protection pp. 333. (in Polish)

Piechocki, A. (1981). Modern and sub-fossil snails (Mollusca) Holly Cross Mountains, Acta Universitatis Lodziensis, 177. (in Polish)

Riedel, A. (1988). Gastropoda terrestria. Catologus faunae Poloniae, 36, 1, pp. 3-316. (in Polish)

Simpson, J.E., Slade, E., Riutta T. \& Taylor, M.E. (2012). Factors affecting soil fauna feeding activity in a fragmented lowland temperature deciduous woodland, PLOS ONE 7(1), e29616. doi.10.1371/journal.pone.0029616.

Świercz, A. (1995). Characteristics rendzinas soils and the Tilio-Carpinetum in Monitoring Station Malik Hill, Biblioteka Monitoringu Środowiska, pp. 47-64. (in Polish)

Świercz, A. (1997). The effect of alkalic emission on soils and pine forest in "Białe Zagtębie", PAN-Oddział w Krakowie, KTN, Kielce 1997. (in Polish)

Świercz, A. (2005). Analysis of soil processes and vegetation transformations in the alkalized forestial habitats in the Świetokrzyski Region, PAN, Komitet „Człowiek i Środowisko”, Zeszyty Naukowe, 39, Warszawa-Kielce 2005.

Świercz, A. (2006). Suitability of pine bark to evaluate pollution caused by cement-lime dust, Journal of Forest Science, 52, pp. $93-98$.

Świercz, A. (2010). Soils cover and use [In:] A Monograph of the Chęcińsko-Kielecki Landscape Park, UJK, Kielce, pp. 93-117. (in Polish)

Tervahattu, H., Lodenius, M. \& Tulisalo, E. (2001). Effects of the reduction of cement plant pollution on foliar and bark chemical composition of Scots pine, Boreal Environment Research, 6, pp. 251-259.

\section{Długotrwały wpływ antropogemicznej alkalizacji na bioróżnorodność mięczaków w obszarze oddziaływania przemysłu cementowo-wapienniczego (południowo-centralna Polska)}

Streszczenie: Prezentowana praca przedstawia wyniki wieloletnich badań malakologicznych i gleboznawczych, które zostały przeprowadzone w zróżnicowanych biotopach (las, bór, łąka) znajdujących się do $2 \mathrm{~km}$ od emitora pyłów, Cementowni Dyckerhoff Sitkówka-Nowiny w latach: 1992-1993 oraz 2008-2009. Celem pracy było przedstawienie zmian właściwości fizyczno-chemicznych gleb, przekształconych pod wpływem alkalizacji, które zadecydowały o składzie gatunkowym malakofauny bytującej w 3 różnych biotopach. 